\title{
Lung ultrasound as a part of cardiological assessment of physically active person after COVID-19
}

\author{
Maria Binkiewicz-Orluk ${ }^{1}$ (D), Marcin Konopka ${ }^{1}$, Agnieszka Jakubiak ${ }^{2}$, Wojciech Król ${ }^{1}$, \\ Marek Kuch ${ }^{2}$, Wojciech Braksator ${ }^{1}$ \\ ${ }^{1}$ Department of Sports Cardiology and Noninvasive Cardiovascular Imaging, Medical University of Warsaw, Warsaw, Poland \\ ${ }^{2}$ Department of Cardiology, Hypertension and Internal Diseases, Medical University of Warsaw, Poland
}

\begin{abstract}
Both the ongoing severe acute respiratory syndrome-related coronavirus 2 pandemic and increasing number of survivors after coronavirus disease 2019 (COVID-19) state new challenges. One of them is proper assessment after infection in order to safe return to professional, physical and social activity and avoidance of undiagnosed, potentially life-threating complications. Significant element is a choice of diagnostic methods for evaluation of the parenchymal changes within the lungs. Despite high resolution computed tomography is still a gold standard, the use of lung ultrasonography is still increasing. It is a complementary diagnostic method which might be performed simultaneously with echocardiography. We present the proposition of use lung ultrasound in a 34-year-old patient, elite athletes- trainer after COVID-19, who developed parenchymal lung changes and recovered from them in a short time interval.
\end{abstract}

Key words: lung ultrasonography, COVID-19, post-COVID complications, sports medicine

Folia Cardiologica 2022; 17, 1: 54-57

\section{Case report}

A 34-year-old physically active male coach of competitive athletes was admitted to the hospital for evaluation after coronavirus disease 2019 (COVID-19) disease.

Medical history of the patient in question during COVID-19 infection: muscle and joint pain, weakness, fever, cough (> 14 days), dyspnoea, reduced saturation to $90 \%$, chest tightness. The patient was treated on an outpatient basis and took antipyretics.

He was assessed on day 23 after a positive polymerase chain reaction (PCR) test - good overall clinical condition, without dyspnoea or stenocardia. Vital signs were normal, electrocardiogram (ECG) revealed sinus bradycardia with a heart rate of $52 / \mathrm{min}$, ECG recording was normal for a physically active person. Laboratory tests revealed leukopenia -3.75 thousand/ $\mu$ l (neutrophil count in blood smear: 1.51 thousand/ $\mu \mathrm{l})$, increased activity of both alanine transaminase (ALT) - $199 \mathrm{U} / \mathrm{I}$ and aspartate transaminase (AST) - $60 \mathrm{U} / \mathrm{I}$, elevated ferritin levels - $361.1 \mathrm{ng} / \mathrm{mL}$; elevated levels of N-terminal pro-B-type natriuretic peptide (NT-proBNP), D-dimers and high-sensitivity cardiac troponin; the results of $\mathrm{C}$-reactive protein (CRP) and other tests were normal. The patient's serum had anti- severe acute respiratory syndrome-related coronavirus 2 (anti-SARS CoV-2) antibodies, IgM 29.68 S/C (positive values for $\geq 1.4 \mathrm{~S} / \mathrm{C}$ ), and IgG $8.20 \mathrm{~S} / \mathrm{C}$ (positive values for $\geq 1 \mathrm{~S} / \mathrm{C}$ ). The echocardiogram revealed normal geometry and proportion of cardiac chambers, normal systolic and diastolic function of both ventricles, normal valvular apparatus, a trace of pericardial fluid. In the 24-hour Holter monitoring, the mean heart rate

Adress for correspondence: Maria Binkiewicz-Orluk MD, Klinika Kardiologii, Nadciśnienia Tętniczego i Chorób Wewnętrznych, ul. Kondratowicza 8, 03-242 Warszawa, Poland, phone +48 2232655 05, e-mail: maria.binkiewicz@gmail.com

This article is available in open access under Creative Common Attribution-Non-Commercial-No Derivatives 4.0 International (CC BY-NC-ND 4.0) license, allowing to download articles and share them with others as long as they credit the authors and the publisher, but without permission to change them in any way or use them commercially. 


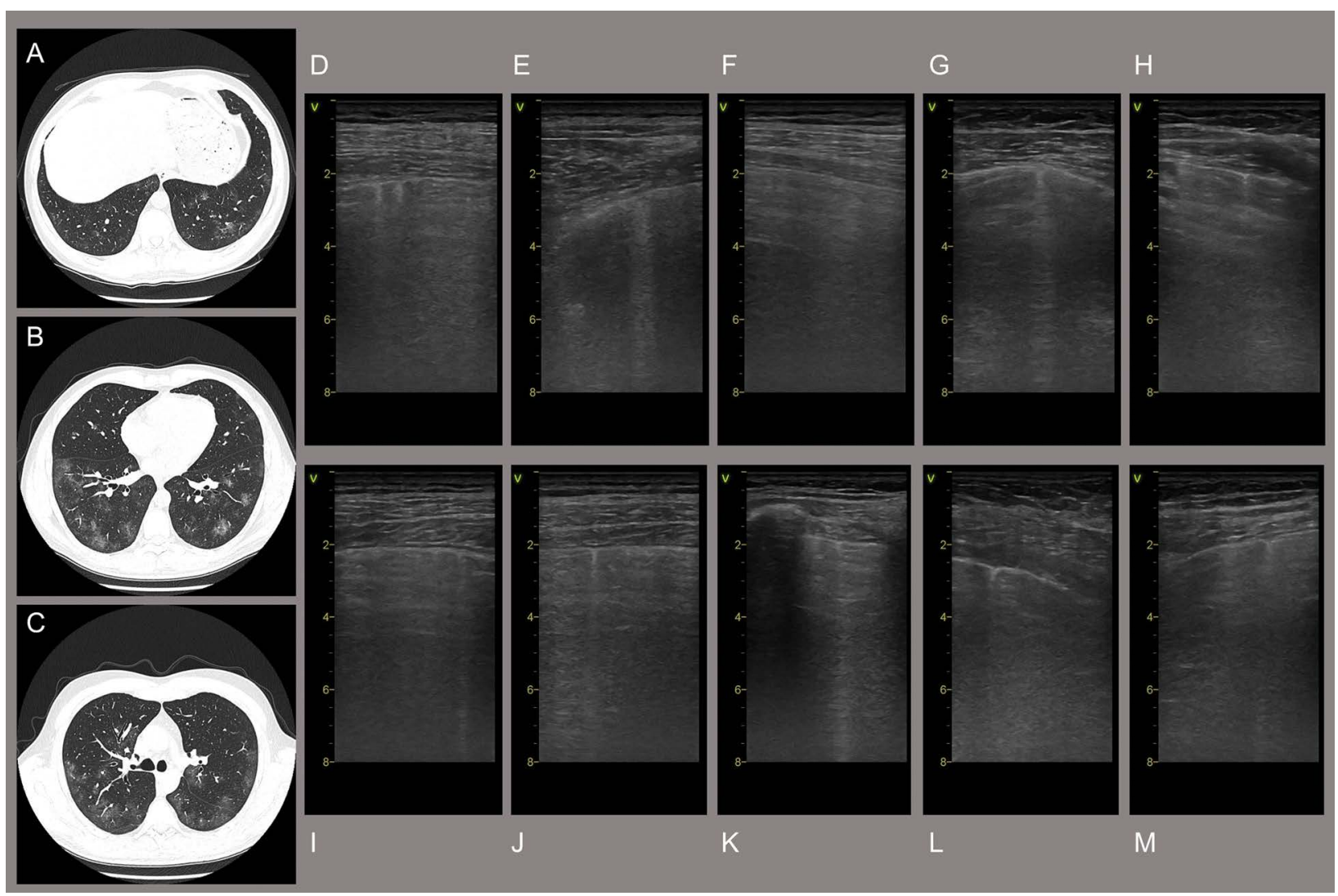

Figure 1. The chest imaging performed on day 23 after a positive polymerase chain reaction (PCR) test result, using different modalities: A-C. The high-resolution computed tomography (HRCT) of the chest revealed bilateral diffuse areas of ground glass opacities, occupying approximately $30 \%$ of the lung parenchyma; D-M. Lung ultrasound (LUS) found 1) changes in pleural line: irregularities (E, H, I, L, M) and interruption of continuity (L, M), 2) small subpleural consolidations accompanied by C lines (E, G, I-K), 3) A-line artifacts (F)

was $66 /$ min, minimum 44/min, maximum 120/min. No arrhythmia was recorded.

Lung ultrasound (LUS) revealed several abnormalities in most areas of the lungs. These abnormalities included 1) pleural line irregularity and interruption of continuity, 2) multiple, small, subpleural inflammatory consolidations accompanied by $\mathrm{C}$ lines (Figure 1 ).

The high-resolution computed tomography (HRCT) revealed areas of ground glass opacities, occupying approx. $30 \%$ of the lung parenchyma. Moreover, there was a $3 \mathrm{~mm}$ nodule in the right lung apex and small adhesions in both lung apices above the diaphragm. In addition, the presence of pericardial fluid (up to $10 \mathrm{~mm}$ ) was proved, which corresponded to the echocardiographic picture.

Because of abnormalities found in laboratory tests and imaging tests, a well-balanced lifestyle, breathing exercises, and self-monitoring were recommended.

The patient was reevaluated after less than 11 weeks. Laboratory tests revealed mild leukopenia (3.88 thousand/ $/ \mu \mathrm{L}$ ) with normal smear; significant reduction in the activity of aminotransferases (ALT $67 \mathrm{U} / \mathrm{L}$, AST $24 \mathrm{U} / \mathrm{L}$ ); normalisation of ferritin levels, without abnormalities in other tests.
Positive IgM (4.72 S/C) and IgG (7.32 S/C) antibody titers were preserved. Echocardiography (echocardiogram) did not reveal the previously present pericardial fluid.

LUS revealed significant regression of previously found lesions - the predominance of A-line artifacts indicating normal lung aeration, which corresponded to the follow-up HRCT - areas of ground glass opacities were found only in a small area of the right lung lower lobe (Figure 2).

To complete the evaluation, an exercise test was performed according to the Bruce protocol - the test was completed when the maximum heart rate was reached at a load of 16.5 metabolic equivalents (METs) and fatigue was $9 / 10$ points on the modified Borg scale, which proved very good exercise tolerance.

The patient returned to full work capacity and physical activity.

\section{Discussion}

Another challenge posed by the SARS-CoV-2 pandemic is to make recovery, i.e. return to work capacity and physical activity, safe. 


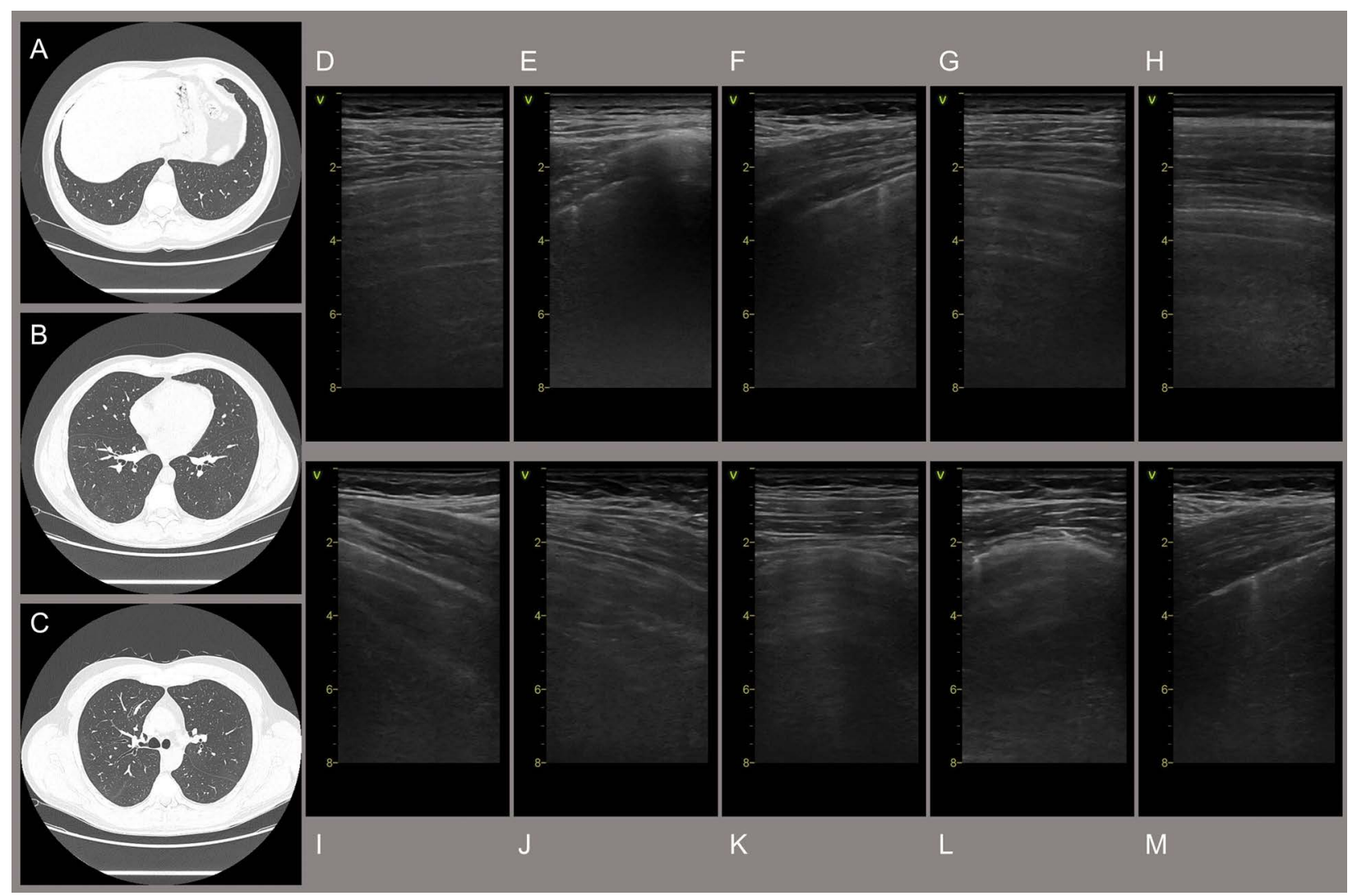

Figure 2. The chest imaging performed on day 99 after a positive polymerase chain reaction (PCR) test result, using different modalities: A-C. The high-resolution computed tomography (HRCT) of the chest revealed significant regression of lesions compared to the previous examinations: lung parenchyma without significant consolidations; D-M. Lung ultrasound (LUS) revealed significant regression of lesions. The following abnormalities were found: 1) A-line artifacts (D, G, H, I-K), 2) irregularity of the pleural line (E, F, L, M) and its small defects (E, F), 3) small subpleural consolidations accompanied by $C$ lines (F, L, M)

In athletes, the assessment of the cardiovascular system is particularly crucial for early diagnosis of life-threatening complications (pulmonary embolism, acute coronary syndromes, arrhythmias, myocarditis, pericarditis or heart failure) [1]. Cardiological assessment methods can be found in the literature in the form of return to play protocols [2]. Although they differ slightly, each method recommends a cardiological assessment in symptomatic patients before their return to intensive training; procedures depend on the duration and severity of symptoms. American guidelines recommend laboratory tests including troponin testing, electrocardiogram, and echocardiogram; depending on obtained results, other tests such as cardiac magnetic resonance (CMR) should be considered. In other protocols, there are suggestions for performing 24-hour Holter monitoring and exercise tests, including spiroergometry. In view of the fact that there are no standardised guidelines, individual decisions are of great importance.

In the reported case, several cardiological examinations (laboratory tests, echocardiography, Holter monitoring, exercise tests) were performed, in which no significant abnormalities were found.

Concerns about the development of pulmonary fibrosis as a residual form of interstitial lesions are reflected in the experience of previous epidemics caused by coronaviruses such as severe acute respiratory syndrome (SARS) and Midle East respiratory syndrome (MERS) [3]. There are data that HRCT lesions that are typical of COVID-19 disease (ground glass opacities, cobblestone pattern, inflammatory consolidations, streaky subpleural lesions) persist in up to $77 \%$ of individuals at 2 months and in $63 \%$ at 3 months after the infection [4].

The accessibility of COVID-19-specific lesions for ultrasound, lack of exposure to ionizing radiation, relatively high sensitivity, reproducibility, the possibility of performing point-of-care examinations, and rapid learning curve have contributed to a significantly increased interest in LUS [5]. From a cardiological point of view, an additional advantage is the possibility of a rapid screening assessment of the lung parenchyma during echocardiography. This method for the assessment of active COVID-19 in hospitalised patients 
has been well established in the literature [6]. There are promising results regarding the use of LUS in the assessment of lesion evolution for the diagnosis of interstitial lung disease (ILD) and other complications - according to some authors, LUS should be the first-line test [5].

Typical ultrasound changes in COVID-19 disease include irregular and/or interrupted pleural line, B-line artifacts, and inflammatory consolidations (smaller, subpleural consolidations accompanied by $\mathrm{C}$ lines, or larger, lobular consolidations). During the recovery phase, there is a gradual regression of the above-mentioned abnormalities, as demonstrated by an increase in the number of areas with A-line artifacts that reflect a properly aerated lung parenchyma [5], which was proved in the reported case.

\section{Conclusions}

The assessment of patients after COVID-19 is reasonable because of the risk of complications involving various organs and systems. Cardiac screening can be performed using available return to play protocols. Both HRCT and LUS are appropriate methods to assess the evolution of interstitial lesions after COVID-19.

\section{Conflict of interest}

The authors declare no conflicts of interest.

\section{Funding}

None.

\section{References}

1. Madjid M, Safavi-Naeini P, Solomon SD, et al. Potential effects of coronaviruses on the cardiovascular system: a review. JAMA Cardiol. 2020; 5(7): 831-840, doi: 10.1001/jamacardio.2020.1286, indexed in Pubmed: 32219363.

2. Phelan $\mathrm{D}$, Kim JH, Chung $\mathrm{EH}$. A game plan for the resumption of sport and exercise after coronavirus disease 2019 (COVID-19) infection. JAMA Cardiol. 2020; 5(10): 1085-1086, doi: 10.1001/jamacardio.2020.2136, indexed in Pubmed: 32402054.

3. Chan KS, Zheng JP, Mok YW, et al. SARS: prognosis, outcome and sequelae. Respirology. 2003; 8(Suppl): S36-S40, doi: 10.1046/j.1440-1843.2003.00522.x, indexed in Pubmed: 15018132.

4. Sonnweber T, Sahanic S, Pizzini A, et al. Cardiopulmonary recovery after COVID-19: an observational prospective multicentre trial. Eur Respir J. 2020; 57(4): 2003481, doi: 10.1183/13993003.034812020.

5. Peixoto AO, Costa RM, Uzun R, et al. Applicability of lung ultrasound in COVID-19 diagnosis and evaluation of the disease progression: a systematic review. Pulmonology. 2021; 27(6): 529-562, doi: 10.1016/j. pulmoe.2021.02.004, indexed in Pubmed: 33931378.

6. Treanor L, Islam N, Ebrahimzadeh S, et al. The cochrane systematic review on thoracic imaging tests for the diagnosis of COVID-19. Radiology. 2021; 299(3): E289, doi: 10.1148/radiol.2021210564, indexed in Pubmed: 33754831. 